\title{
Revisión sistemática sobre factores de riesgo asociados a artralgia persistente en el paciente con fiebre del chikunguña
}

Risk Factors Associated With Persistent Arthralgia In Chikungunya Virus-Infected Patients - A Systematic Review

Recepción: 31/10/2016 | Aceptación: 18/01/2018

\author{
Édgar Yaset Caicedo-Ochoa \\ Universidad Pedagógica y Tecnológica de Colombia , \\ Colombia \\ Jorge Andrés UrRutia-Gómez \\ Universidad Pedagógica y Tecnológica de Colombia, \\ Colombia \\ Daniel Sebastián Fernández-Niño ${ }^{\mathrm{a}}$ \\ Universidad Pedagógica y Tecnológica de Colombia, \\ Colombia \\ Yardany Rafael MéndeZ-FAndiño \\ Universidad Pedagógica y Tecnológica de Colombia, \\ Colombia
}

a Correspondencia: grab.uptc@gmail.com

Cómo citar: Caicedo-Ochoa EY, Urrutia-Gómez JA, Fernández-Niño DS, Méndez-Fandiño YR. Revisión sistemática sobre factores de riesgo asociados a artralgia persistente en el paciente con fiebre del chikunguña. Univ. Med. 2018;59(2). doi: https://doi.org/10.11144/Javeriana.umed59-2.chik

\section{RESUMEN}

Antecedente: La fiebre del chikunguña es una enfermedad tropical infecciosa, caracterizada por la aparición de fiebre, erupción cutánea y artralgias, cuya incidencia ha aumentado en los últimos años, en Asia y Latinoamérica. Aunque es una enfermedad con baja mortalidad, se ha relacionado con limitación funcional a largo plazo, por su compromiso articular. Objetivo: Llevar a cabo una revisión sistemática de la literatura sobre los factores de riesgo asociados a artralgia persistente en el paciente con fiebre de chikunguña. Metodología: Se buscó información en bases de datos como PubMed, Embase, ScienceDirect, Scopus y Google Académico, con el descriptor de búsqueda (chikungunya fever OR chikungunya) AND (arthralgia). Resultados: Se obtuvieron 4281 estudios de los cuales al final se seleccionaron seis estudios de cohorte por su similitud metodológica en la evaluación de factores asociados y de los que se obtuvieron diferentes resultados en el análisis de datos. Conclusión: No existe información concluyente acerca de factores modificables que permitan predecir la persistencia de artralgias en pacientes infectados por el virus de la fiebre del chikunguña. Hacen falta estudios que permitan evaluar la presencia de dichos factores, fin de disminuir la morbilidad asociada a la artralgia persistente.

Palabras clave

fiebre chikunguña; artralgia; factores de riesgo.

\section{ABSTRACT}

Background: Chikungunya fever is an infectious tropical disease characterized by the onset of fever, rash and arthralgia, whose incidence has increased in recent years in Asia and Latin America. Although 
it is a disease with low mortality, it has been associated with long-term functional limitation, by their joint involvement. Objective: To conduct a systematic review of the literature on risk factors associated with persistent arthralgia in patients with Chikungunya fever (CHKF). Methods: A search for information in databases such as Pubmed, Embase, ScienceDirect, Scopus and Google Scholar was performed, search the descriptor (OR chikungunya fever chikungunya) AND (arthralgia). Results: 4281 studies at the end of which six cohort studies for methodological similarity were selected in the evaluation of associated factors, obtaining different results in the analysis were obtained. Conclusion: There is no conclusive information about modifiable factors that predict persistent joint pain in patients infected CHKV. Studies are needed to assess the presence of these factors to decrease the morbidity associated with persistent arthralgia.

Keywords

chikungunya fever; arthralgia; chikungunya virus; risk factors.

\section{Introduccion}

El virus del chikunguña (CHIKV) es un miembro del género de los Alphavirus, perteneciente a la familia Togaviridae (1). Dentro de los alfavirus que producen enfermedad al ser humano hay dos grupos filogenéticos distintos clasificados según su distribución geográfica: uno caracterizado por causar fiebre, erupción cutánea y artralgias, donde se incluye el CHIKV, presente principalmente en el viejo mundo, y otro, caracterizado por causar encefalitis, presente en el nuevo mundo, en este grupo se encuentra el virus de la encefalitis equina del oeste (2). El CHIKV fue aislado por primera vez en África occidental, donde se bautizó con el nombre chikungunya, el cual hace referencia a la postura encorvada que se desarrolla en un individuo como consecuencia de los síntomas articulares y proviene de la lengua bantú del pueblo Makonde, ubicado Tanzania y Mozambique (3).

El CHIKV mantiene su ciclo natural por transmisión entre un artrópodo vector y un huésped vertebrado (4); en el vector, un mosquito de la familia Aedes produce una infección prolongada con efectos mínimos en las funciones biológicas normales (5). El mosquito existe en climas tropicales en zonas urbanas y suburbanas (6). Después de la picadura del mosquito al huésped, el CHIKV induce una respuesta febril aguda, conocida como fiebre del chikunguña, acompañada de artralgias (7) que pueden durar varios meses. Se considera que esta fiebre, probablemente, ha ocurrido en África por siglos, con brotes exportados a Asia y América en los siglos XVIII y XIX (8).

La historia de la fiebre del chikunguña cambió con la aparición en el 2004 del linaje del océano Índico, originado en África, que se extendió en el 2005 a las islas del océano Índico, donde se produjeron brotes con altas tasas de prevalencia. El mejor estudiado ha sido el de la isla francesa de Reunión, con cerca de 300.000 (9). Desde finales del 2013, una cepa de CHIKV procedente de Asia llegó al Caribe y se ha extendido por todo el continente americano, principalmente Centro y Suramérica (10).

Desde el primer reporte de la fiebre de chikunguña, en Tanganica, en 1952 (11), se han descrito casos en distintas ciudades de África y Asia (12). Durante los últimos 30 años se han identificado epidemias en India, Islandia, Hong Kong y Malasia $(12,13,14)$, con brotes en distintas islas del océano Índico, principalmente en la isla de Reunión. En Europa se han presentado casos de transmisión autóctona, debido a viajeros infectados con viremias importantes y que podrían eventualmente establecer patrones endémicos (15).

Para la epidemia actual en la región de las Américas, el primer caso confirmado se reportó en el 2013, en la isla franco-holandesa de Saint Martin, en el mar Caribe (16). A partir de este, comenzaron a generarse los primeros reportes de caso en el continente. Según la Organización Mundial de la Salud para las Américas, para la semana epidemiológica (SE) 42, actualizada hasta el 21 de octubre del 2016, se habían reportado un total de 107.570 casos confirmados de transmisión autóctona en el continente; así mismo, se había reportado un total de 106 fallecidos. La zona del continente con menor número de casos reportados pertenece al Cono Sur, y dentro de este, Chile no había registrado ningún caso de transmisión autóctona (17).

En Colombia se reportó el primer caso importado el 15 de julio de 2014, en Cali, correspondiente a una mujer proveniente de 
República Dominicana, y el 11 de septiembre del mismo año se reportó el primer caso correspondiente a transmisión autóctona en el municipio de San Joaquín (Bolívar) (18). El Instituto Nacional de Salud, en su último boletín, SE 41 del 2016, reportó un total de 19.157 para ese año (19).

La fase aguda de la enfermedad puede durar desde un par de días a dos semanas con posterior resolución del cuadro. Luego inicia un periodo de incubación posterior a la picadura, que puede durar de uno a doce días (20). Esta fase se caracteriza por presentar fiebre elevada $(>38,5$ $\left.{ }^{\circ} \mathrm{C}\right)$ de inicio súbito, acompañada de dolor articular o muscular generalizado que puede ser de intensidad moderada a severa; además, en algunos casos se pueden presentar erupciones cutáneas tipo mácula o pápula en extremidades, tronco y cara; o síntomas inespecíficos como cefalea y fotofobia $(21,22)$.

Es de aclarar que, por su gravedad, la poliartralgia puede ser incapacitante hasta en un $60 \%$ de los casos, y puede ser simétrica y periférica, con principal afectación de dedos, muñecas, tobillos, codos, rodillas y artejos (23). En algunos casos es posible que haya hinchazón sinovial como síntoma acompañante; otros síntomas menos comunes son los digestivos (24). El riesgo de complicaciones a largo plazo es bajo; aun así se ven cuadros con complicaciones neurológicas como encefalitis, mielopatías, neuropatías periféricas y miopatías (25). Algunos casos atípicos se pueden presentar sin sintomatología asociada (26).

Según la Organización Mundial de la Salud, el diagnóstico de la enfermedad se sospecha por el cuadro clínico presentado (fiebre > 38,5 ${ }^{\circ} \mathrm{C}$ y artralgia severa), además de la guía por datos epidemiológicos de la región o de viajes previos. Entre los métodos para confirmar la infección se encuentran el cultivo vírico, la reacción en cadena de polimerasa (PCR) con transcriptasa inversa para detectar anticuerpos de inmonoglubulina (IgM) antiCHKV, detección de anticuerpos IgM por Elisa, o la demostración de anticuerpos IgG específicos en aumento (27).
En la mayoría de los casos, la artralgia se resuelve por completo; pero algunos pacientes tienen el riesgo de presentar un síndrome de artritis crónica, el cual es una continuación de la fase aguda y puede durar de meses a años. Afecta comúnmente las mismas articulaciones de la fase aguda, pero con menor severidad (28). Esta progresión crónica de la enfermedad es debida a una inflamación erosiva articular (29). Se han descrito algunos factores asociados a la artralgia a largo plazo en estudios de cohorte, como la edad del paciente o la carga viral, pero no hay revisiones que concluyan los principales desencadenantes de la persistencia de este síntoma $(30,31)$.

Por lo anterior, es necesario saber cuáles factores se asocian a la persistencia del dolor articular en pacientes que sufrieron la enfermedad, el cual es el objetivo del presente estudio: indagar cuáles son los principales factores relacionados con la persistencia de los dolores articulares en estos pacientes.

\section{Metodología}

El desarrollo del trabajo siguió las recomendaciones de la guía de Preferred Reporting Items for Systematic Reviews and Metaanalyses (32).

Búsqueda de literatura. Se indagó en las bases de datos de PubMed, Embase, ScienceDirect, Scopus y Google Académico, para registros relevantes de antes del 27 de agosto del 2015, utilizando el esquema de búsqueda: (chikungunya fever OR chikungunya) AND arthralgia. Teniendo en cuenta que se realizó una búsqueda exploratoria, donde los números de trabajos relacionados a CHKV es poca, el esquema tiene características para aumentar su sensibilidad. No se realizaron filtros adicionales o restricciones de lenguaje.

Selección de estudios. Se buscaron estudios de tipo ensayos clínicos, cohortes y casos y controles. Los criterios de inclusión fueron: paciente con infección por $\mathrm{CHKV}$, descripción de síntomas articulares, seguimiento del paciente y descripción de factores de riesgo asociados 
a la cronicidad de los síntomas articulares. Los criterios de exclusión fueron: reportes de casos, series de casos y cartas al editor. La búsqueda se hizo por parte de los investigadores (EY Caicedo, JA Urrutia y DS Fernández) a los cuales se les asignaron las bases de datos de manera aleatoria para una evaluación independiente. Se valoraron todos los registros resultantes de la búsqueda, tanto su título como su resumen, en los cuales se describían pacientes con CHKV y artralgia persistente. Los registros que presentaban discrepancias fueron evaluados por un revisor externo (YR Méndez).

Luego se evaluaron los documentos obtenidos durante la búsqueda, de los cuales se registró la siguiente información: titulo del estudio, autor principal, año, resumen, verificación de criterios de inclusión, objetivo del estudio, tipo de estudio, temporalidad, lugar donde se realizó, método diagnóstico de $\mathrm{CHKV}$, fuente de la información de los pacientes, criterios de inclusión y exclusión, identificación y definición de las variables de resultado del estudio, datos descriptivos de la población y factores de riesgo presentados. La evaluación de riesgo de sesgos de los estudios se hizo mediante la escala de Newcastle-Ottawa (33).

Análisis de la información. La presente revisión no cuenta con los lineamientos en metodología o igualdad metodológica para el abordaje de un metanálisis, por lo cual se realizó un análisis cualitativo de la información en marco de la revisión sistemática.

\section{Resultados}

\section{Búsqueda}

Se obtuvieron 4281 registros de la búsqueda, de los cuales se seleccionaron 33 estudios que en el título y el resumen presentaban los criterios de inclusión. Al realizar la evaluación del texto en extenso, se seleccionaron únicamente seis estudios que cumplían con la descripción de cohorte y artralgia persistente en el contexto crónico de paciente con fiebre de chikunguña (figura 1). No obstante, la calidad de los estudios y de las variables en cada uno de ellos solo presentaban como variable de factor de riesgo en común el sexo femenino, por lo cual el grupo de autores decidió no realizar un metanálisis de la información. En lugar de esto se planteó un análisis cualitativo de los estudios encontrados.

Figura 1.Flujograma de búsqueda de la información

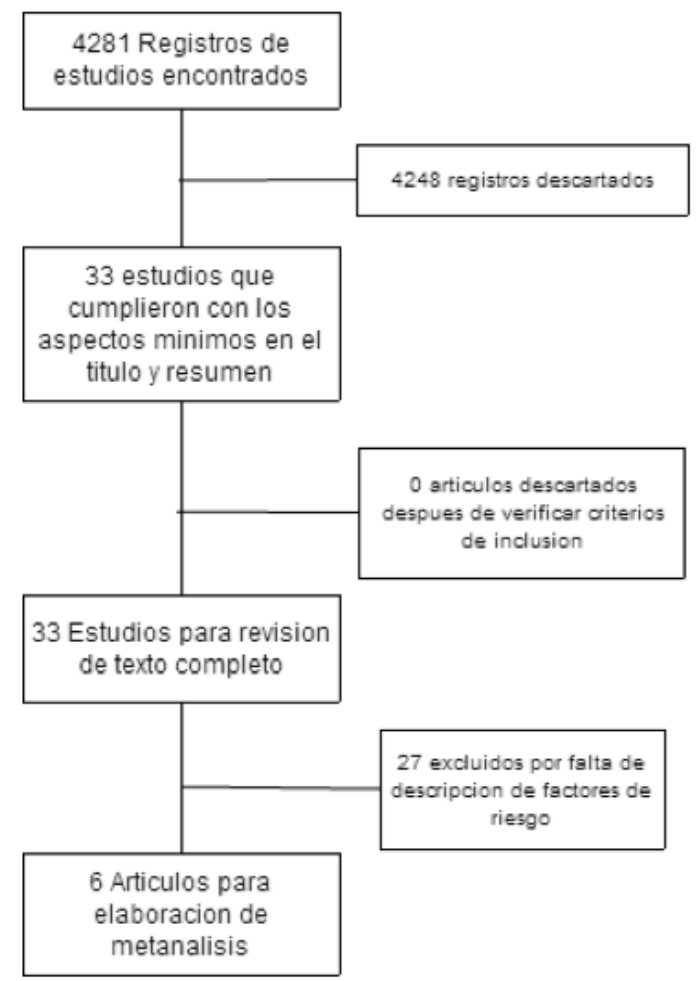

Los estudios encontrados y las variables estudiadas como factores de riesgo más relevantes se muestran en la tabla 1 .

Tabla 1.Resultado de los estudios seleccionados

\begin{tabular}{|c|c|c|c|c|c|c|c|}
\hline Referencia & $\begin{array}{l}\text { Metodo de } \\
\text { diagnostico } \\
\text { de CHKV }\end{array}$ & Lugar & $\begin{array}{l}\text { Tiempo } \\
\text { del estudio }\end{array}$ & $\begin{array}{l}\text { Número } \\
\text { de } \\
\text { pacientes }\end{array}$ & $\begin{array}{l}\text { Cronicidad } \\
\text { de artralgas }\end{array}$ & Factores de riesgo descritos & 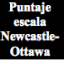 \\
\hline Moro, 2012 & $\begin{array}{l}\operatorname{IgMe~IgG~} \\
\text { anti-CHKV } \\
\text { PCR RT }\end{array}$ & Italia & $\begin{array}{l}\text { Julio 2007- } \\
\text { agosto } \\
2008\end{array}$ & 250 & 12 moses & 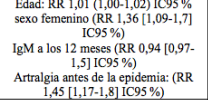 & *** \\
\hline $\begin{array}{l}\text { Larrieu, } \\
2010\end{array}$ & $\begin{array}{l}\text { IgM- IgG } \\
\text { anti-CHKV } \\
\text { PCR RT }\end{array}$ & Francia & $\begin{array}{c}\text { Marzo } \\
\text { 2005- } \\
\text { diciembre } \\
2007 \\
\end{array}$ & 29 & $18-36$ meses & 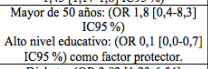 & ** \\
\hline $\begin{array}{l}\text { Schilte, } \\
2013\end{array}$ & $\begin{array}{l}\text { IgMc IgG } \\
\text { antit-CHKV } \\
\text { PCR RT }\end{array}$ & Francia & $\begin{array}{c}\text { Marzo } \\
2005 \text {-mayo } \\
2006\end{array}$ & 87 & 14 meses & 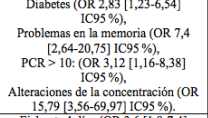 & *** \\
\hline $\begin{array}{l}\text { Ramachand } \\
\text { ran, } 2014\end{array}$ & $\begin{array}{l}\text { Triada } \\
\text { clinica }\end{array}$ & India & $\begin{array}{c}\text { Mayo } \\
2006 \text {-junio } \\
2006\end{array}$ & 81 & 15 dias & 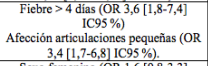 & ** \\
\hline $\begin{array}{c}\text { Sissoko. } \\
2009\end{array}$ & $\begin{array}{l}\text { Ig M e IgG } \\
\text { anti-CHKV } \\
\text { PCR RT }\end{array}$ & $\begin{array}{l}\text { Isla de } \\
\text { Reunión }\end{array}$ & 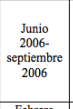 & 147 & 15 meses & 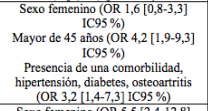 & ** \\
\hline $\begin{array}{l}\text { Essackjee, } \\
2013\end{array}$ & $\begin{array}{l}\text { Tríada } \\
\text { cllnica }\end{array}$ & Mauricio & \begin{tabular}{|c|} 
Febrero \\
$2006-$ \\
noviembre \\
2006
\end{tabular} & 173 & 27 meses & 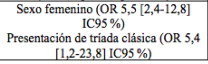 & ** \\
\hline
\end{tabular}




\section{Estudios}

Moro y colaboradores (26) evaluaron 250 pacientes, de los cuales casi el $50 \%$ eran mayores de 60 años, y el 54\%, mujeres. Con el seguimiento a 154 pacientes, los autores reportaron, al menos, una condición crónica previa a la infección. De estos, 89 pacientes ya referían algún tipo de enfermedad articular, seguidas de otras condiciones como enfermedad cardiaca, cáncer y diabetes. La cohorte evaluó a los sujetos en dos momentos: síntomas a los 4-5 meses y 12-13 meses. Al momento de 4-5 meses, 180 pacientes presentaban artralgia en las siguientes articulaciones: $69 \%$ en manos, $58,3 \%$ en pies y $57,8 \%$ en tobillos. No obstante, dentro de este grupo de pacientes se evidenció que presentaban diagnósticos concomitantes como síndrome artrálgico, artritis, tenosinovitis y fibromialgia, factores de confusión que pueden presentarse concomitante al desarrollo de la enfermedad. El estudio describe como factor de riesgo edad, sexo femenino, IgM positiva a los 4-5 meses, IgM positiva a los 12-13 meses y artralgia antes de la epidemia. Sin embargo, ninguno es estadísticamente significativo.

Larrieu y colaboradores (30) evaluaron 42 pacientes, quienes tuvieron en promedio una edad de 51 años. El $60 \%$ eran hombres. Se estudiaron a un plazo de 18-36 meses, de los cuales tan solo 17 todavía presentaban molestias articulares, con ubicación en tobillos (41\%), manos y pies $(29 \%)$ y rodillas $(29 \%)$. Siete pacientes referían presentar signos de inflamación. Los factores de riesgo presentados fueron: mayor de 50 años, nivel educativo alto y presencia de alguna comorbilidad durante la fase aguda sobrepeso. El único de estos factores relevante es el nivel educativo alto.

Schitle y colaboradores (34) definieron el seguimiento a los 4, 6, 14 y 36 meses después del evento agudo. La cohorte tuvo a 180 pacientes, de los cuales 129 sujetos a los 4 meses todavía referían artralgia persistente; 122, a los 6 meses; 148 , a los 14 meses, y 102, a los 36 meses. Las articulaciones que presentaron de forma más frecuente la artralgia persistente fueron dedos, muñecas, rodillas y tobillos. Asociados a otros síntomas locales de inflamación, síntomas cutáneos, mialgia y dolor osteo-ligamentoso. Los factores de riesgo que se presentaron fueron cuantificación viral mayor 100.000 copias/ml, PCR mayor a 10, diabetes mellitus, enfermedades de la memoria y trastornos en la concentración. Es de resaltar la posible relación con los niveles de PCR y la tendencia a la cronicidad.

Ramachandran y colaboradores (35) hicieron el seguimiento de 403 pacientes; el $40 \%$ hombres con una edad promedio de 37,7 años. Este estudio resaltó especialmente los factores sociales sobre el impacto y el desarrollo de la fiebre de chikunguña, relacionando la no educación o educación apenas de nivel primario con un ingreso familiar en promedio al mes de 66,5 dólares y las condiciones de vivienda precarias. El 40\% de los pacientes al mes referían artralgia persistente; el $16 \%$, a $1-3$ meses; el $12,7 \%$, a 3-6 meses, y el 7\%, y a más de un año. Las articulaciones más afectadas fueron rodillas (96\%), muñecas (80\%), falanges (77\%), entre otras. Los factores de riesgo presentados fueron: edad mayor de 35 años, fiebre por 4 días o más y afección de articulaciones pequeñas. Ninguno de estos factores de riesgo presentó significancia estadística.

Sissoko y colaboradores (36) estudiaron una cohorte de 147 sujetos, en que el $31 \%$ eran hombres con un promedio de edad de 52 años. Los síntomas articulares en su localización más frecuente fueron tobillos (76\%), muñecas $(62 \%)$ y rodillas $(44 \%)$. En el seguimiento se observó que 63 pacientes todavía presentaban persistencia de los síntomas. Los factores de riesgo que describió el estudio fueron: mayor de 45 años, sexo femenino y presencia de enfermedad concomitante como osteoartrosis. Estas variables significativas de mayoría de edad y de osteoartrosis son eventos que se relacionan con una mayor intensidad de la sintomatología articular en los pacientes, por lo cual podrían dar lugar a confusiones respecto a la sensación de dolor articular propia de la fiebre del chikunguña.

Essackjee y colaboradores (37) tomaron un total de 173 pacientes con diagnóstico clínico, de los cuales el 79\% reportaron síntomas musculo- 
esqueléticos. En el estudio también aludieron a la existencia de políticas que restringen el acceso a las pruebas inmunológicas para la detección de CHKV. Los factores de riesgo relevantes fueron sexo femenino y la presentación de la tríada fiebre, erupción cutánea y artralgia.

\section{Discusión}

La artralgia persistente puede ser la complicación más frecuente e incapacitante de la infección por CHKV, y dada su importancia epidémica en el tercer mundo, es un problema de salud pública que impacta las frágiles economías de estos países (8). Sin embargo, los estudios disponibles para este análisis están relacionados con características poblacionales durante el brote de CHKV, muchos de ellos sin una rigurosidad metodológica que permita responder la pregunta de investigación de este estudio. La definición de caso probable y confirmado no fue clara y en muchos de ellos el diagnóstico fue clínico sin confirmación bioquímica $(35,37)$.

Las series de casos reportadas coinciden en la persistencia de los síntomas a lo largo del tiempo en evaluaciones periódicas a los 4 y 12 meses (26), con una frecuencia mayor en las articulaciones de las manos. En otra cohorte, el $56 \%$ de los pacientes persistieron con síntomas articulares a los 36 meses (34). El sexo femenino fue un factor asociado en varias de las series, incluso en estudios en los cuales la distribución de hombres fue de un $60 \%$ $(30,36)$. El sexo femenino, adicionalmente, ha sido reportado en otros estudios como factor asociado a artralgia persistente (38). La edad mayor a 50 años también estuvo asociada con la presencia de artralgias durante el seguimiento a un año $(26,30,34,36)$. No hay claridad acerca del comportamiento de la variable artralgia previa a la infección CHKV, ya que puede ser un factor de confusión en la interpretación del cuadro clínico o definitivamente empeorar la severidad de la artralgia luego de la infección (26). Es de considerar la relación presente en algunos estudios entre problemas en la memoria y la concentración, con artralgia persistente, sea una variable de confusión por relacionarse estas condiciones a alteraciones en la calidad de vida del paciente y su percepción de dolor (26).

Respecto a los marcadores de laboratorio, la persistencia de IgM positiva durante el seguimiento, incluso hasta los 12 meses, carga viral mayor a 100.000 copias $/ \mathrm{ml}$ y PCR mayor a 10 constituyen factores relacionados con la persistencia de la artralgia. De ahí que sean necesarios estudios de mayor envergadura para demostrar si estos valores de laboratorio podrían comportarse como predictores de artralgia persistente en un futuro estudio $(26,34,39)$.

Otras variables de tipo socioeconómico se relacionaron con artralgia persistente, por ejemplo: nivel educativo bajo, condiciones de vivienda e ingresos económicos mensuales, sin que ello fuera estadísticamente significativo, debido a que estas condiciones generalmente se vinculan con condiciones de vivienda poco higiénicas que pueden promover la diseminación de la enfermedad. Sin embargo, esta interpretación es riesgosa, ya que no hay una relación como tal de este fenómeno con la cronicidad de los síntomas $(30,35)$.

Las limitaciones metodológicas referidas no permiten sintetizar la información de una forma homogénea o realizar un análisis cuantitativo más profundo; pero permiten establecer algunos vínculos con artralgia persistente que podrían evaluarse con nuevos estudios para determinar factores de riesgo modificables asociados a artralgia persistente en pacientes infectados por el virus.

\section{Conclusiones}

Los factores asociados con la aparición de artralgia persistente en pacientes con fiebre del chikunguña no se encuentran claramente descritos, pues los estudios de cohortes presentan una marcada heterogeneidad metodológica. El sexo femenino es el factor común de los estudios analizados, relacionado con la persistencia de los síntomas articulares; no obstante, los resultados de los estos no presentan uniformidad. 
Son necesarias nuevas investigaciones con mayor rigurosidad metodológica sobre el seguimiento de los pacientes con manifestaciones articulares desde la fase aguda para determinar la relación de estas manifestaciones con la cronicidad de la artralgia y la limitación funcional en pacientes con infección por CHKV.

\section{Referencias}

1. Couderc T, Lecuit M. Chikungunya virus pathogenesis: From bedside to bench. Antiviral Res. 2015 Sep;121:120-31.

2. Powers AM, Brault AC, Shirako Y, Strauss EG, Kang W, Strauss JH, et al. Evolutionary relationships and systematics of the alphaviruses. J Virol. 2001 Nov;75(21):10118-31.

3. Robinson MC. An epidemic of virus disease in Southern Province, Tanganyika territory, in 1952-1953. Trans R Soc Trop Med Hyg. 1955 Jan 1;49(1):28-32.

4. Strauss JH, Strauss EG. The alphaviruses: gene expression, replication, and evolution. Microbiol Rev. 1994 Sep;58(3):491-562.

5. Jose J, Snyder JE, Kuhn RJ. A structural and functional perspective of alphavirus replication and assembly. Future Microbiol. 2009 Sep;4:837-56.

6. Thiberville S-D, Moyen N, Dupuis-Maguiraga L, Nougairede A, Gould EA, Roques P, et al. Chikungunya fever: Epidemiology, clinical syndrome, pathogenesis and therapy. Antiviral Res. 2013 Sep;99(3):345-70.

7. Suhrbier A, Jaffar-Bandjee M-C, Gasque P. Arthritogenic alphaviruses-an overview. Nat Rev Rheumatol. 2012 Jul;8(7):420-9.

8. Halstead SB. Reappearance of Chikungunya, Formerly Called Dengue, in the Americas. Emerg Infect Dis [Internet]. 2015 Apr [citado 2016 oct 25];21(4). Disponible en: http://wwwnc.cdc.gov/eid/article/21/4/ 14-1723_article.htm.

9. Gérardin $\bar{P}$, Guernier V, Perrau J, Fianu A, Le Roux K, Grivard P, et al. Estimating Chikungunya prevalence in $\mathrm{La}$ Réunion Island outbreak by serosurveys:
Two methods for two critical times of the epidemic. BMC Infect Dis. 2008 Jul 28;8:99.

10. Weaver SC, Lecuit M. Chikungunya virus and the global spread of a mosquitoborne disease. N Engl J Med. 2015 Mar 26;372(13):1231-9.

11. Pialoux G, Gaüzère B-A, Jauréguiberry S, Strobel M. Chikungunya, an epidemic arbovirosis. Lancet Infect Dis. 2007 May;7(5):319-27.

12. Mohan A, Kiran DHN, Manohar IC, Kumar DP. Epidemiology, clinical manifestations, and diagnosis of Chikungunya fever: lessons learned from the re-emerging epidemic. Indian J Dermatol. 2010 Jan;55(1):54-63.

13. Mavalankar D, Shastri P, Raman P. Chikungunya epidemic in India: A major public-health disaster. Lancet Infect Dis. 2007 May;7(5):306-7.

14. Bhatia R, Narain JP. Re-emerging chikungunya fever: some lessons from Asia. Trop Med Int Health. 2009 Aug $1 ; 14(8): 940-6$.

15. Team EC for DP and C(ECDC)-HCU-E editorial. An outbreak of chikungunya fever in the province of Ravenna, Italy. European Centre for Disease Prevention and Control(ECDC) - Health Comunication Unit; 2007.

16. Van Bortel W, Dorleans F, Rosine J, Blateau A, Rousset D, Matheus S, et al. Chikungunya outbreak in the Caribbean region, December 2013 to March 2014, and the significance for Europe. Euro Surveill [Internet]. 2014;19(13). Disponible en: http://www.eurosurveillance.org/conte nt/10.2807/1560-7917.ES2014.19.13.2075 9\#html_fulltext

17. Organización Panamericana de la Salud, Organización Mundial de la Salud. Chikunguña: datos, mapas y estadísticas. Semana epidemiológica/SE 42. 21 de octubre de 2016. 2016 [citado 2016 oct 25]. Disponible en: http://www.paho.org/hq/index.php?opt ion $=$ com_topics $\&$ view $=$ readall $\&$ cid $=59$ $32 \&$ Itemid $=40931$ \&lang $=$ es. 
Édgar Yaset Caicedo-Ochoa, Jorge Andrés Urrutia-Gómez, Daniel Sebastián Fernández-Niño et al.

18. Durán M, Gómez S. Chikunguña en Colombia, el inicio de la transmisión autóctona, 2014. Inf Quinc Epidemiológico Nac [Internet]. 2014;19(18). Disponible en: http://www.ins.gov.co/iqen/IQUEN/IQ EN\%20vol\%2019\%202014\%20num\%201 8.pdf.

19. Instituto Nacional de Salud. Boletín Epidemiológico Semanal [Internet]. 2016 [citado 2016 oct 25];(41). Disponible en: http://www.ins.gov.co/boletin-epidemi ologico/Boletn $\% 20$ Epidemiolgico/2015\%2 OBoletin\%20epidemiologico\%20semana\% 2041.pdf.

20. Burt FJ, Rolph MS, Rulli NE, Mahalingam S, Heise MT. Chikungunya: a reemerging virus. The Lancet. 2012 Feb;379(9816):662-71.

21. Borgherini G, Poubeau P, Staikowsky F, Lory M, Moullec NL, Becquart JP, et al. Outbreak of Chikungunya on Reunion Island: Early clinical and laboratory features in 157 adult patients. Clin Infect Dis. 2007 Jun 1;44(11):1401-7.

22. Lo Presti A, Lai A, Cella E, Zehender G, Ciccozzi M. Chikungunya virus, epidemiology, clinics and phylogenesis: A review. Asian Pac J Trop Med. 2014 Dec $1 ; 7(12): 925-32$.

23. Rezza G, Nicoletti L, Angelini R, Romi R, Finarelli A, Panning M, et al. Infection with chikungunya virus in Italy: an outbreak in a temperate region. The Lancet. 2007 Dec 7;370(9602):1840-6.

24. Paul BJ, Pannarkady G, Moni SP, Thachil EJ. Clinical profile and long-term sequelae of Chikungunya fever. Indian J Rheumatol. 2011 Mar 1;6(1):12-9.

25. Daginawala H, Chandak N, Kashyap R, Kabra D, Karandikar P, Saha S, et al. Neurological complications of Chikungunya virus infection. Neurol India. 2009;57(2):177.

26. Moro ML, Grilli E, Corvetta A, Silvi G, Angelini R, Mascella F, et al. Long-term chikungunya infection clinical manifestations after an outbreak in Italy: A prognostic cohort study. J Infect. 2012 Aug 1;65(2):165-72.

27. World Health Organisation. Guidelines on clinical management of chikunguña fever [Internet]. 2008 [citado 2016 oct 25]. Disponible en: http://www.wpro.who.int/mvp/topics/n td/Clinical_Mgnt_Chikungunya_WHO_S EARO.pdf.

28. de Andrade DC, Jean S, Clavelou P, Dallel R, Bouhassira D. Chronic pain associated with the Chikungunya Fever: long lasting burden of an acute illness. BMC Infect Dis. 2010;10:31.

29. Manimunda SP, Vijayachari P, Uppoor R, Sugunan AP, Singh SS, Rai SK, et al. Clinical progression of chikungunya fever during acute and chronic arthritic stages and the changes in joint morphology as revealed by imaging. Trans $\mathrm{R}$ Soc Trop Med Hyg. 2010 Jun;104(6):392-9.

30. Larrieu S, Pouderoux N, Pistone T, Filleul L, Receveur M-C, Sissoko D, et al. Factors associated with persistence of arthralgia among chikungunya virusinfected travellers: Report of 42 French cases. J Clin Virol. 2010 Jan 1;47(1):85-8.

31. Borgherini G, Poubeau P, Jossaume A, Gouix A, Cotte L, Michault A, et al. Persistent arthralgia associated with chikungunya virus: a study of 88 adult patients on reunion island. Clin Infect Dis Off Publ Infect Dis Soc Am. 2008;47(4):469-75.

32. Liberati A, Altman DG, Tetzlaff J, Mulrow C, Gotzsche PC, Ioannidis JPA, et al. The PRISMA statement for reporting systematic reviews and metaanalyses of studies that evaluate healthcare interventions: explanation and elaboration. BMJ. 2009 Dec 4;339(jul21 1):b2700b2700.

33. Wells G, Shea B, O'Connell D, Peterson J, Welch V, Losos M, et al. The NewcastleOttawa Scale(NOS) for assessing the quality of nonrandomised studies in metaanalyses [Internet]. [Citado 2016 oct 25]. Disponible en: http://www.ohri.ca/program s/clinical_epidemiology/oxford.asp 
34. Schilte C, Staikovsky F, Couderc T, Madec Y, Carpentier F, Kassab S, et al. Chikungunya virus-associated longterm arthralgia: a 36-month prospective longitudinal study. PLOS Negl Trop Dis. 2013 Mar 21;7(3):e2137.

35. Ramachandran V, Kaur P, Kanagasabai K, Vadivoo S, Murhekar M. Persistent arthralgia among Chikungunya patients and associated risk factors in Chennai, South India. J Postgrad Med. 2014;60(1):3.

36. Sissoko D, Malvy D, Ezzedine K, Renault P, Moscetti F, Ledrans M, et al. Postepidemic Chikungunya disease on Reunion Island: Course of rheumatic manifestations and associated factors over a 15 -month period. PLOS Negl Trop Dis. 2009 Mar 10;3(3):e389.

37. Essackjee K, Goorah S, Ramchurn SK, Cheeneebash J, Walker-Bone K. Prevalence of and risk factors for chronic arthralgia and rheumatoid-like polyarthritis more than 2 years after infection with chikungunya virus. Postgrad Med J. 2013 Aug;89(1054):440-7.

38. Win MK, Chow A, Dimatatac F, Go CJ, Leo YS. Chikungunya fever in Singapore: Acute clinical and laboratory features, and factors associated with persistent arthralgia. J Clin Virol. 2010 Oct 1;49(2):111-4.

39. Chopra A, Anuradha V, Ghorpade R, Saluja M. Acute Chikungunya and persistent musculoskeletal pain following the 2006 Indian epidemic: a 2-year prospective rural community study. Epidemiol Amp Infect. 2012 May;140(5):842-50. 